\title{
GMR
}

\section{Inhibition of miR-660-5p expression suppresses tumor development and metastasis in human breast cancer}

\author{
Y. Shen ${ }^{1}$, Y.F. Ye' ${ }^{2}$ L.W. Ruan ${ }^{3}$, L. Bao ${ }^{1}$, M.W. Wu ${ }^{1}$ and Y. Zhou ${ }^{1}$ \\ ${ }^{1}$ Shaoxing Women \& Children's Hospital, Shaoxing, China \\ ${ }^{2}$ Department of Clinical Laboratory, \\ The First Affiliated Hospital of Zhengzhou University, Zhengzhou, China \\ ${ }^{3}$ Department of Breast, Shaoxing People's Hospital, \\ Shaoxing Hospital of Zhejiang University, Shaoxing, China \\ Corresponding author: Y. Zhou \\ E-mail: Zhouying305@163.com \\ Genet. Mol. Res. 16 (1): gmr16019479 \\ Received October 26, 2016 \\ Accepted December 20, 2016 \\ Published February 23, 2017 \\ DOI http://dx.doi.org/10.4238/gmr16019479 \\ Copyright $(2017$ The Authors. This is an open-access article distributed under the terms of \\ the Creative Commons Attribution ShareAlike (CC BY-SA) 4.0 License.
}

\begin{abstract}
Breast cancer, which derives from the epithelium of the mammary glands, is one of the most common cancers diagnosed in women globally. To date, the authors of many studies have reported that the deregulation of microRNAs (miRNAs) plays a crucial role in the occurrence, development, and metastasis of tumors. Here, we discovered that miR-660-5p was upregulated in the breast cancer cell lines MCF7 and MDA-MB-231 compared with that in the normal control cell line CCD-1095Sk. We then inhibited the expression of miR-660-5p to investigate its biological function in cancer development, progression, and metastasis. We determined the changes in the levels of expression of transcription factor $\mathrm{CP} 2$ (TFCP2) and CDKN1A to further clarify the specific mechanism involved. The results showed that downregulation of miR-660-5p significantly suppressed the proliferation, migration, and invasion of MCF7 breast cancer cell. Moreover, inhibition of miR-
\end{abstract}

Genetics and Molecular Research 16 (1): gmr16019479 
660-5p promoted cell cycle G1 arrest and reduced apoptosis in breast cancer cells. The specific mechanism studies confirmed that TFCP2 was a direct downstream target of miR-660-5p. Aberrant expression of miR-660-5p repressed TFCP2 expression, whereas inhibition of miR660-5p decreased TFCP2 protein expression, which is a vital factor in the downstream signaling pathway. In conclusion, miR-660-5p can regulate the proliferation, migration, and invasion of human breast cancer cells, and is a novel potential therapeutic target for the clinical treatment of breast cancer.

Key words: miR-660-5p; Breast cancer; Cell proliferation; Apoptosis; Cell migration

\section{INTRODUCTION}

Breast cancer is one of the most common cancers diagnosed in women globally, and is the second leading cause of cancer-related death among women, after lung cancer (Siegel et al., 2013; DeSantis et al., 2014). Furthermore, metastasis is the most common cause of mortality in patients with breast cancer. Breast cancer is a heterogenous disease characterized by diverse molecular subtypes, various clinical behaviors, and distinct molecular features (Sørlie et al., 2001; Goldhirsch et al., 2011). Previous studies have disclosed that the development of malignant tumors in the breasts is a complex multistep process that involves numerous genetic alterations, giving rise to the deregulation of significant complicated pathways in cellular processes, such as migration, proliferation, apoptosis, and invasion (Porter et al., 2001; Bourguignon et al., 2003; Umetani et al., 2006). MicroRNAs (miRNAs) are non-coding RNA molecules that are approximately 18-25 nucleotides long. They modulate the expression of target genes and play an essential role in the biological and pathological processes of diseases (Friedman et al., 2009). The use of miRNAs as potential biomarkers for diagnosis and prognosis and as therapeutic agents in various cancers has risen in recent years (Liu et al., 2012). Several studies have indicated that miRNAs, such as miR-181a and miR-34a, serve as oncogenes or tumor suppressors; they affect breast cancer development and metastasis by regulating mRNA targets (He et al., 2005; Lee and Dutta, 2007; Li et al., 2013; Taylor et al., 2013).

Transcription factor CP2 (TFCP2), also known as LSF and LBP-1c, was first identified as a late transcriptional activator of the simian virus 40 (SV40) promoter in HeLa cells (Kim et al., 1987). Subsequently, it was found that TFCP2 is expressed in all mammalian cell types as a ubiquitous transcription factor, and plays a role in cell cycle progression and cell survival, as well as in cell lineage-specific functions (Xu et al., 2015). Previous studies have also shown that $T F C P 2$ plays a critical role in malignant cancer progression from the G1 phase to the $\mathrm{S}$ phase of the cell cycle (Saxena et al., 2010). TFCP2 functions as an oncogene in hepatocellular carcinomas, and an increased expression level of TFCP2 promotes malignant progression (Saxena et al., 2010). Goto et al. (2016) found an opposite role of TFCP2 in melanoma, whereby increased TFCP 2 expression levels suppressed both the anchorage-dependent and -independent growth of melanoma cells. However, the functions and the specific molecular mechanism of action of TFCP 2 in breast cancer remain obscure.

Previous studies have revealed that miRNA-660-5p (miR-660-5p) is upregulated in patients with breast cancer (Krishnan et al., 2015). Several databases, such as TARGETSCAN,

Genetics and Molecular Research 16 (1): gmr16019479 
MIRDB, and MICRORNA.ORG, have shown that TFCP2 is a potential target of miR-660-5p. In the present study, we investigated the expression of miR-660-5p in two breast cancer cell lines and a normal cell line to confirm its upregulation in breast cancer cells. We then inhibited the expression of miR-660-5p to investigate its biological function in cancer development and progression. The changes in the levels of expression of TFCP 2 and CDKN1A were determined to further clarify the specific mechanism of miR-660-5p.

\section{MATERIAL AND METHODS}

\section{Cell lines and cell culture}

The human breast cancer cell lines MCF7 and MDA-MB-231, and the normal human breast fibroblast cell line CCD-1095Sk were purchased from the American Type Culture Collection (ATCC, Manassas, VA, USA). The human embryonic kidney cell line HEK293 was also from ATCC. MDA-MB-231 cells were cultured in L15 medium, and the MCF7, CCD1095Sk, and HEK293 cells were cultured in Dulbecco's modified Eagle's medium (DMEM; Gibco, Waltham, MA, USA) supplemented with 10\% fetal bovine serum (FBS).

\section{RNA isolation and reverse transcription polymerase chain reaction (RT-PCR) analysis}

The total RNAs of the various cell lines were extracted using TRIzol reagent (Invitrogen, Carlsbad, CA, USA) according to the manufacturer instructions. The resulting RNA molecules were reverse-transcribed using a specific miRNA primer (Hsa-miR-660$5 p$; Funeng Biotechnology Co., Ltd., Guangzhou, China) for miRNA analysis, and were then subjected to RT-PCR, following the manufacturer protocols for the miScript SYBR Green PCR kit and the miScript Reverse Transcription kit (Funeng Biotechnology Co., Ltd., Guangzhou, China).

\section{Cell transfection}

The miR-660-5p inhibitor and the negative control miRNA inhibitor were synthesized by Guangzhou Ruibo Biotechnology (Guangzhou, China). Either the miR-660-5p inhibitor or the negative control miRNA was transfected into the MCF7 cells using Lipofectamine 2000 transfection reagent, according to the manufacturer protocol (Invitrogen). MCF7 cells transfected with only Lipofectamine 2000 transfection reagent (Invitrogen) were used as a MOCK group. Three groups of transfected MCF7 cells were used for further analysis and all assays were conducted with three replicates.

\section{Cell proliferation determination}

The 3-(4,5-dimethylthiazol-2-yl)-2,5-diphenyltetrazolium bromide (MTT) assay was used to measure the cell proliferation rates in the three groups of transfected MCF7 cells. The cells were seeded onto 96-well plates at a density of 4000 cells per well. The cells were then maintained in a culture medium containing 5\% FBS for $48 \mathrm{~h}$. Finally, the absorbance at 490 nm was measured.

Genetics and Molecular Research 16 (1): gmr16019479 


\section{Cell cycle and cell apoptosis analysis}

A propidium iodide cell cycle detection kit (Biotechnology's Beyotime Institute, Beijing, China) was used to analyze the cell cycle on a FACSCalibur flow cytometer (BD Biosciences, NY, USA). An Annexin V-phycoerythrin (PE) Apoptosis Detection kit I (BD) was used to perform the apoptosis assay, according to the manufacturer instructions. The results were also analyzed using a FACSCalibur flow cytometer.

\section{Cell migration and invasion assays}

The cells were seeded onto $35-\mathrm{mm}$ dishes that had been coated with fibronectin to carry out the wound-healing migration assay. Once the cells had reached $100 \%$ confluence, a scratch (approximately $500 \mu \mathrm{m}$ long) was created on the confluent monolayer using a sterile p200 pipette tip. The cellular debris was then removed by replacing the medium with fresh serum-free medium. During the subsequent 48 -h culture of the cells, we obtained images at 0,24 , and $48 \mathrm{~h}$. We used a Matrigel Transwell chamber (BD) filled with serum-free medium for the invasion assay. A total of $1 \times 10^{4}$ cells were filled into the upper chamber. Medium containing 10\% FBS served as the chemoattractant in the lower chamber. After incubating the chamber at $37^{\circ} \mathrm{C}$ for $64 \mathrm{~h}$, the cells that were adhered to the lower membrane were fixed, stained, and counted using a microscope and a counting chamber (Olympus, Tokyo, Japan).

\section{RT-PCR analysis and western blotting}

The mRNA and protein expression levels of TFCP2 and CDKN1A were determined to investigate the mechanism of action of miR-660-5p. For mRNA analysis, RNA was reverse-transcribed with Moloney murine leukemia virus reverse transcriptase (Promega, Madison, WI, USA) and random primers (Promega). SYBR Premix Ex Taq II (Takara Biotechnology Co., Ltd., China) was used for RT-PCR. The PCR primers for TFCP2 were: F: 5'-TCTGGCCGACGAAGTGATTG-3'; and R: 5'-ATCAGGAGGCAAACTCGACTC-3'. The PCR primers for CDKN1A were: F: 5'-TGTCCGTCAGAACCCATG-3'; and R: 5'-59TGGGAAGGTAGAGCTTGG-3'. For western blotting, the cell cytoplasm and nucleus lysates were separated by sodium dodecyl sulfate polyacrylamide gel electrophoresis, and analyzed on polyvinylidene fluoride membranes (Millipore, USA). Rabbit anti-human polyclonal TFCP2 or CDKN1A (dilution, 1:1000; Sigma-Aldrich, St. Louis, MO, USA) and monoclonal mouse anti-human $\beta$-actin (dilution, 1:5000; Sigma-Aldrich) antibodies were incubated with the blotted membranes. Secondary horseradish peroxidase-conjugated goat anti-rabbit IgG antibody (dilution, 1:5000; Santa Cruz Biotechnology, Inc. Santa Cruz, CA, USA) was also incubated with the membranes. An Enhanced Chemiluminescence System (Pierce Biotechnology, Inc., Rockford, IL, USA) was used to analyze the protein bands. Glyceraldehyde 3-phosphate dehydrogenase (GAPDH) and proliferating cell nuclear antigen (PCNA) expression levels were used as internal normalization controls.

\section{Dual-luciferase reporter assay}

XhoI/NotI-digested psiCHECK-2 vectors (Promega) were used for the DualLuciferase reporter assay. A fragment of the 3'-UTR (untranslated region) of the TFCP2 gene

Genetics and Molecular Research 16 (1): gmr16019479 
containing the miR-660-5p target sequence (CAAGGAT) was inserted into the vector. Either an miR-660-5p mimic or a negative control miRNA was co-transfected into the HEK293 cells using Lipofectamine 2000 transfection reagent (Invitrogen), according to the manufacturer instructions. After incubation for $48 \mathrm{~h}$, the cells were lysed. A Dual-Luciferase Reporter Assay kit (Promega) was used to carry out the luciferase reporter assay.

\section{Statistical analysis}

All experiments were performed in triplicate. The data were analyzed using SPSS 14 software (SPSS, Chicago, Illinois) and are expressed as the mean \pm SD. Unless otherwise mentioned, two-sided Student's $t$-tests or one-way analysis of variance (ANOVA) were used to determine differences between groups. $\mathrm{P}<0.05$ was viewed as a significant difference.

\section{RESULTS}

\section{Varying expression of miR-660-5p in breast cancer}

To explore the significance and varying expression of miR-660-5p in breast cancer, mRNA expression arising from miR-660-5p was determined in breast cancer MDA-MB-231 and MCF7 cell lines, and in normal breast fibroblast cell line CCD-1095Sk. As shown in Figure 1, miR-660-5p was significantly upregulated in the breast cancer cell lines, especially in the MCF-7 cell line, in comparison with the CCD-1095Sk cells. To explore the biological function of miR-660-5p in breast cancer cells, miR-660-5p inhibitor was transfected into the MCF7 cells for further investigation.

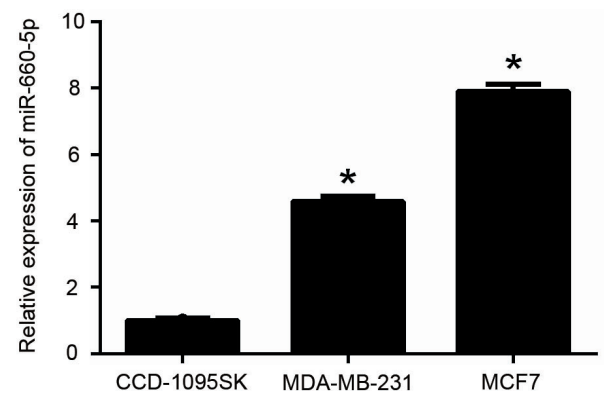

Figure 1. Expression levels of miR-660-5p measured by reverse transcription polymerase chain reaction (RT-PCR) in normal breast fibroblast cells and breast cancer cell lines. $* \mathrm{P}<0.05$.

\section{Effects of miR-660-5p inhibitor on proliferation and the cell cycle in MCF7 cells}

As shown in Figure 2, the results of the MTT assay indicated that downregulation of miR-660-5p significantly inhibited MCF7 cell proliferation. The cell cycle results are shown in Figure 3; the results of the cell cycle distribution analysis by flow cytometry in the three groups of transfected MCF7 cells are shown in Figure 3A, B, and C. The G1 phase comprised $60.2 \%$ anti-miR-660-5p inhibitor-transfected cells, which was significantly higher than the percentage of control cells (Figure 3D). This indicates that the miR-660-5p inhibitortransfected MCF7 cells were arrested in the G1 phase.

Genetics and Molecular Research 16 (1): gmr16019479 


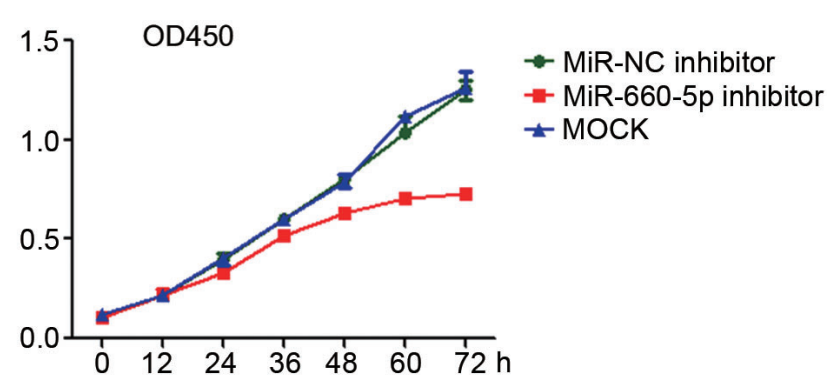

Figure 2. Effects of the miR-660-5p inhibitor on the proliferation of MCF7 cells, as shown by the absorbance at $490 \mathrm{~nm}$.
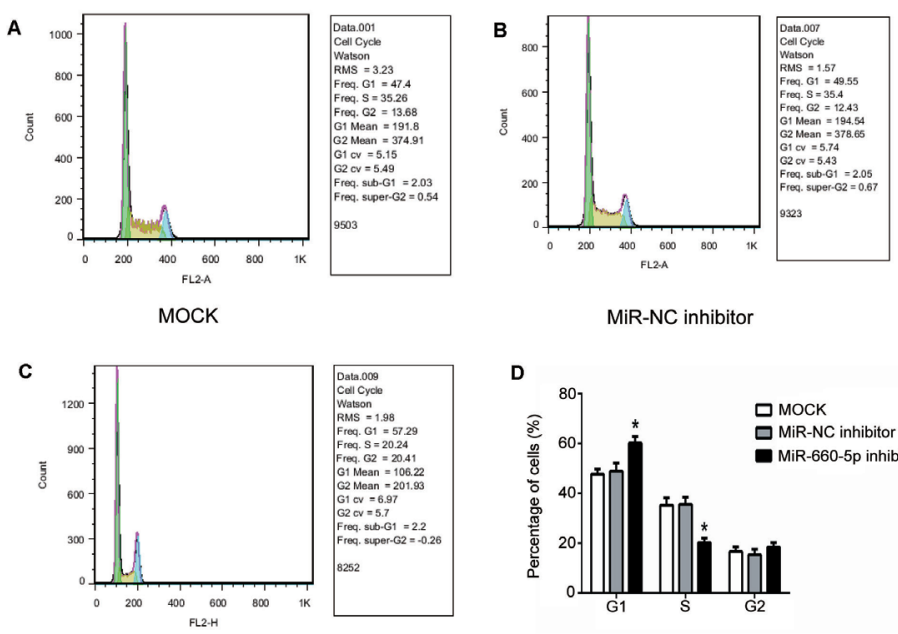

MiR-NC inhibitor

D

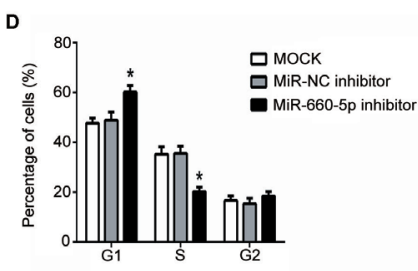

Figure 3. Cell cycle analysis of the transfected MCF7 cells. A. B. and C. Analysis of the cell cycle distribution by flow cytometry in different transfected MCF7 cells. D. The percentage of cells in different cell stages. $* \mathrm{P}<0.05$.

\section{Downregulation of miR-660-5p induced apoptosis in MCF7 cells}

Figure 4 reveals that downregulation of miR-660-5p induced apoptosis in the MCF7 cells. The apoptosis of MCF7 cells transfected with miR-660-5p inhibitor or control miRNA inhibitor was measured by flow cytometry and observed using an electron microscope (Figure 4A and B). The MCF7 cells transfected with miR-660-5p inhibitor had a $19.92 \%$ apoptosis rate, which was significantly higher than the cells in the control group (Figure 4C). The electron microscope images shown in Figure 4B confirm the results.

\section{Inhibition of miR-660-5p reduced the migration and invasion of MCF7 cells}

The results from the wound-healing migration experiment (Figure 5) show that the mobility of the MCF7 cells was significantly reduced after transfection with miR-660-5p inhibitor (Figure 5A). The results of the Transwell assay show that, compared with the control 
cells, the inhibition of miR-660-5p markedly reduced the invasion of MCF7 cells (Figure 5B). These results suggest that miR-660-5p inhibition significantly reduced the migration and invasion of MCF7 cells.
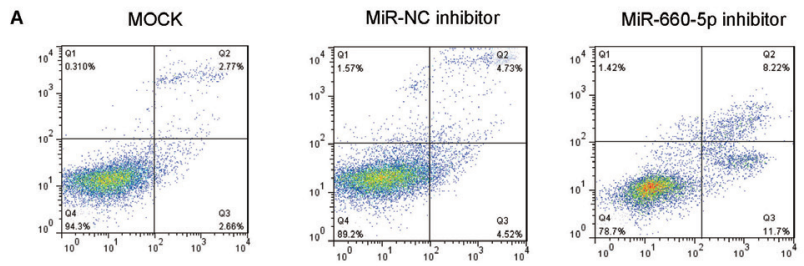

B
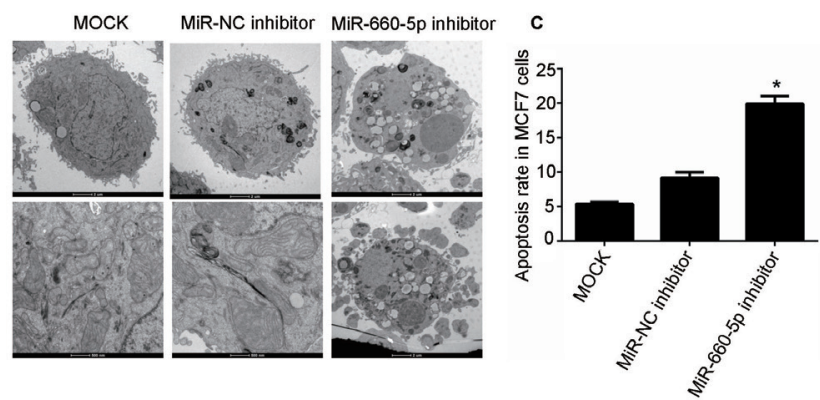

Figure 4. Apoptosis of the transfected MCF7 cells. A. and B. Effect of miR-660-5p downregulation on the apoptosis of MCF7 cells transfected with miR-660-5p inhibitor or control miRNA inhibitor was measured by flow cytometry and visualized using an electron microscope. C. Apoptosis rate of the various transfected MCF7 cells. $* \mathrm{P}<0.05$.

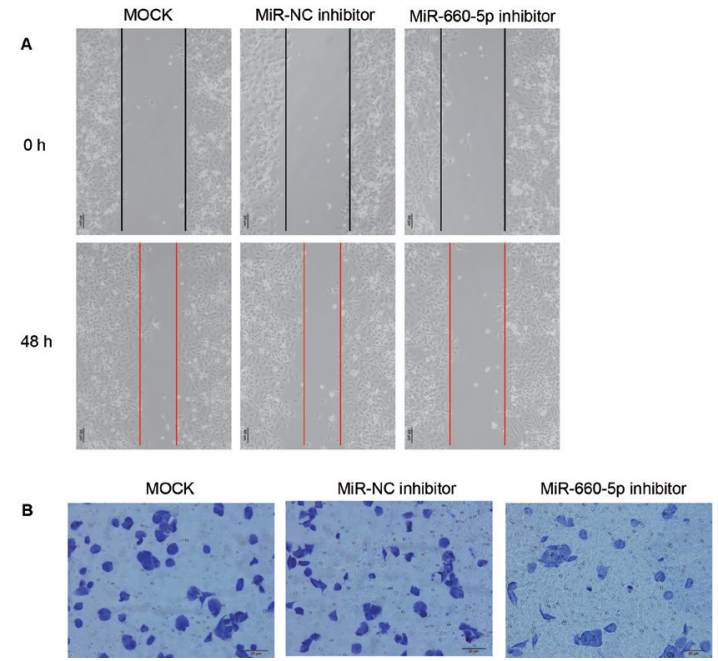

Figure 5. Inhibition effect of the miR-660-5p inhibitor miRNA on the invasion and migration of MCF7 cells. A. Wound-healing assay of MCF7 cells transfected with miR-660-5p inhibitor or control miRNA inhibitor. B. Invasion assay of MCF7 cells transfected with miR-660-5p inhibitor or control miRNA inhibitor.

Genetics and Molecular Research 16 (1): gmr16019479 


\section{TFCP2 was the direct downstream target of miR-660-5p}

The phenotypic data mentioned above indicate that the growth and migration of breast cancer cells were both reduced by the inhibition of miR-660-5p. Moreover, TARGETSCAN, MIRDB, and MICRORNA.ORG were used to predict the targets of miR-660-5p. Interestingly, our results suggest that TFCP 2 is indeed a potential target of miR-660-5p (Figure 6). We found that the 3'-UTR of TFCP2 mRNA contains a site that complements the seed region of miR660-5p (Figure 6A). The human TFCP2 3'-UTR fragments with wild-type (WT 3'-UTR) or mutant (mutated 3'-UTR) miR-660-5p-binding sequences were cloned into the psiCHEK-2 vector (Figure 6A). Figure 6B shows that after miR-660-5p transfection, the relative luciferase activity of the reporter with WT 3'-UTR was significantly reduced. Moreover, this suppression was reversed by the site-directed deletion of the miR-660-5p-binding site of the 3'-UTR of TFCP2 (Figure 6B). The results confirm that miR-660-5p directly binds to this site.

Furthermore, we evaluated the ability of miR-660-5p to regulate TFCP2 expression in human breast cells. The expression levels of TFCP2 were determined in MCF7 cells transfected with the mimic or the inhibitor of miR-660-5p and miR-NC, as shown in Figure $6 \mathrm{C}$ and D. The TFCP 2 mRNA and protein levels were upregulated by the knock-down of miR660-5p in MCF7 cells compared with the miRNA control (Figure 6C and D). Above all, these results explicitly indicate that $T F C P 2$ is a direct target of miR-660-5p in breast cancer cells.

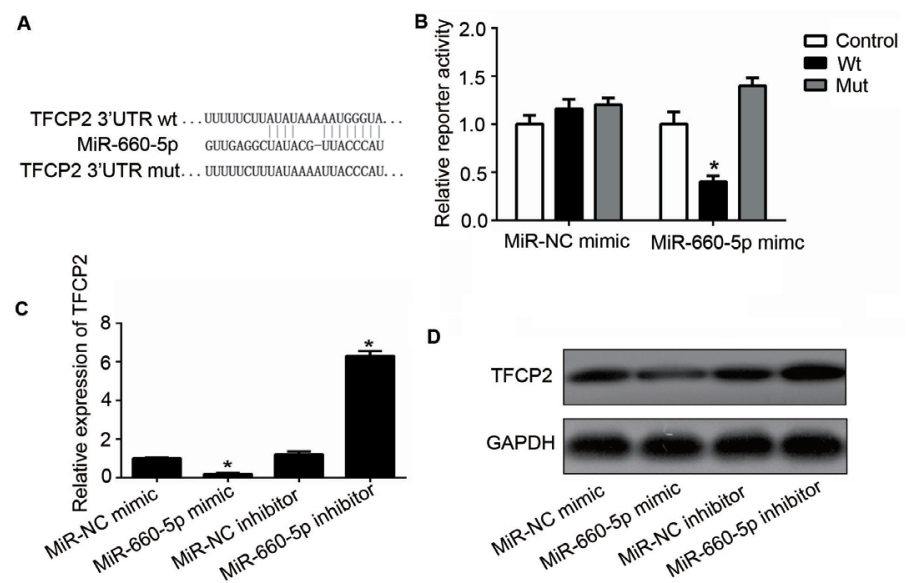

Figure 6. Potential target determination of miR-660-5p in breast cancer cells. A. Sequence alignment between miR$660-5 \mathrm{p}$ and the 3'-UTR of TFCP2. B. The histogram bars represent the relative dual luciferase activity after the transfection of HEK293 cells with miR-660-5p mimics or control miRNAs. ${ }^{*} \mathrm{P}<0.05$ relative to miR-NC. C. and D. The mRNA and protein expression levels of TFCP2 were examined by RT-PCR and western blotting in MCF7 cell transfected with miR-660-5p mimic, control miRNA mimic, miR-660-5p inhibitor, or control miRNA inhibitor.

\section{MiR-660-5p modulated the expression of CDKN1A}

Because previous studies have shown that $T F C P 2$ is a vital factor in the regulation of CDKN1A, we investigated the effect of miR-660-5p on CDKN1A in MCF7 cells. Figure 7 shows that the levels of nuclear CDKN1A mRNA (Figure 7A) and CDKN1A protein (Figure 7B) were significantly upregulated. That is, miR-660-5p promotes the proliferation, migration, and invasion of breast cells by regulating CDKN1A.

Genetics and Molecular Research 16 (1): gmr16019479 
A

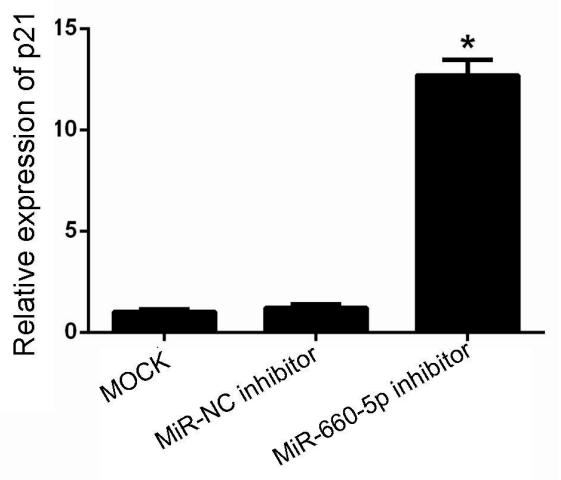

B

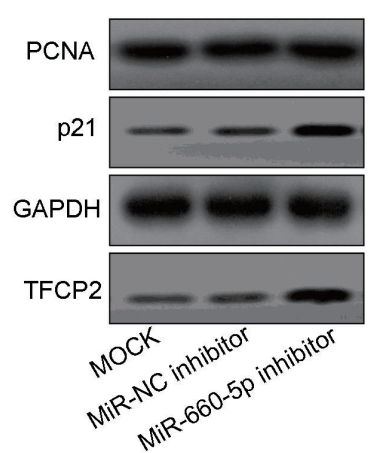

Figure 7. The mRNA and protein expression levels of p21 were examined by reverse transcription polymerase chain reaction (RT-PCR) and western blotting in transfected MCF7 cells. A. The mRNA expression levels of p21. B. The protein expression levels of $\mathrm{p} 21 . * \mathrm{P}<0.05$.

\section{DISCUSSION}

The authors of several studies have reported that the dysregulation of miRNA is involved in tumorigenesis or tumor development, and increasing numbers of authors have suggested that miRNA is a critical factor in tumor cell proliferation, apoptosis, and metastasis. Fortunato et al. (2014) reported that miR-660-5p is downregulated in patients with lung cancer and its replacement inhibits lung tumorigenesis by targeting MDM2-p53 interaction. In contrast, Krishnan et al. (2015) have reported that miR-660-5p is significantly upregulated in patients with breast cancer and can be considered a potential prognostic marker for breast cancer. In the present study, we found that miR-660-5p was significantly upregulated in human breast cancer MDA-MB-231 and MCF7 cell lines. Although the upregulation of miR-660-5p in breast cancer has been described before, the specific mechanism of action of miR-660-5p in breast cancer development and metastasis remains unknown. The present study provides the first evidence that the downregulation of miR-660-5p in breast cancer MCF7 cells significantly inhibits cell proliferation, migration, and invasion, and induces cell cycle arrest in phase G1 and cell apoptosis. Our results indicate that miR-660-5p may be a novel potential oncogene that plays a vital role in the development and metastasis of breast cancer.

As reported, the biological function of miRNAs is to regulate target genes by directly inactivating mRNA or inhibiting protein synthesis (Chekulaeva and Filipowicz, 2009). When we investigated the molecular mechanism of action of miR-660-5p, we found that TFCP2 has a binding site for miR-660-5p within its 3'-UTR region, as predicted. We also found that TFCP2 3'-UTR luciferase reporter activity was reduced by miR-660-5p overexpression, and this reduction was reversed by the knock-down of the miR-660-5p seed binding site. The mRNA and protein levels of TFCP2 were also suppressed by the overexpression of miR-660$5 \mathrm{p}$. The inhibition of miR-660-5p increased the expression level of TFCP2 in breast cancer cells. Therefore, $T F C P 2$ can be considered a direct target of miR-660-5p in breast cancer cells.

However, as mentioned above, TFCP2 has been identified as an oncogene or suppressor gene in various cancers. In the present study, inhibition of miR-660-5p induced the expression of TFCP2 in breast cancer cells, and miR-660-5p negatively regulated TFCP2 expression and function as a suppressor gene in breast cancer cells. Moreover, several studies 
have shown that CDKN1A plays a crucial role in cancer cells by modulating cell cycle arrest, cell growth, proliferation, migration inhibition, and apoptosis induction (Abbas and Dutta, 2009). Goto et al. (2016) found that TFCP2 plays a predominant role in the regulation of CDKN1A, thereby inhibiting cell growth. In our study, CDKN1A expression was significantly upregulated when miR-660-5p was inhibited. Therefore, we conclude that downregulation of miR-660-5p suppresses tumor development and metastasis by targeting TFCP2, which further regulates CDKN1A in human breast cancer.

In conclusion, in the present study we discovered that the inhibition of miR-660-5p suppresses proliferation, migration, and invasion, and induces the apoptosis of human breast cancer cells through an miR-660-5p/TFCP2/CDKN1A signaling pathway. Although the downstream mechanism of the miR-660-5p/TFCP2/CDKN1A signaling pathway and other relevant downstream factors remain obscure, the data from our study suggest that miR-660-5p and its downstream targets may be potential therapeutic targets in human breast cancer.

\section{Conflicts of interest}

The authors declare no conflict of interest.

\section{ACKNOWLEDGMENTS}

Research supported by the Zhejiang Province (Medical Funding Grant \#2012KYB217 and \#2015KYB405) and the Public Projects of Zhejiang Province (Grant \#2016C33223).

\section{REFERENCES}

Abbas T and Dutta A (2009). p21 in cancer: intricate networks and multiple activities. Nat. Rev. Cancer 9: 400-414. http:// dx.doi.org/10.1038/nrc2657

Bourguignon LY, Singleton PA, Zhu H and Diedrich F (2003). Hyaluronan-mediated CD44 interaction with RhoGEF and Rho kinase promotes Grb2-associated binder-1 phosphorylation and phosphatidylinositol 3-kinase signaling leading to cytokine (macrophage-colony stimulating factor) production and breast tumor progression. J. Biol. Chem. 278: 29420-29434. http://dx.doi.org/10.1074/jbc.M301885200

Chekulaeva M and Filipowicz W (2009). Mechanisms of miRNA-mediated post-transcriptional regulation in animal cells. Curr. Opin. Cell Biol. 21: 452-460. http://dx.doi.org/10.1016/j.ceb.2009.04.009

DeSantis C, Ma J, Bryan L and Jemal A (2014). Breast cancer statistics, 2013. CA Cancer J. Clin. 64: 52-62. http://dx.doi. org $/ 10.3322 /$ caac. 21203

Fortunato O, Boeri M, Moro M, Verri C, et al. (2014). Mir-660 is downregulated in lung cancer patients and its replacement inhibits lung tumorigenesis by targeting MDM2-p53 interaction. Cell Death Dis. 5: e1564. http://dx.doi.org/10.1038/ cddis. 2014.507

Friedman RC, Farh KK, Burge CB and Bartel DP (2009). Most mammalian mRNAs are conserved targets of microRNAs. Genome Res. 19: 92-105. http://dx.doi.org/10.1101/gr.082701.108

Goldhirsch A, Wood WC, Coates AS, Gelber RD, et al.; Panel members (2011). Strategies for subtypes - dealing with the diversity of breast cancer: highlights of the St. Gallen International Expert Consensus on the Primary Therapy of Early Breast Cancer 2011. Ann. Oncol. 22: 1736-1747. http://dx.doi.org/10.1093/annonc/mdr304

Goto Y, Yajima I, Kumasaka M, Ohgami N, et al. (2016). Transcription factor LSF (TFCP2) inhibits melanoma growth. Oncotarget 7: 2379-2390.

He L, Thomson JM, Hemann MT, Hernando-Monge E, et al. (2005). A microRNA polycistron as a potential human oncogene. Nature 435: 828-833. http://dx.doi.org/10.1038/nature03552

Kim CH, Heath C, Bertuch A and Hansen U (1987). Specific stimulation of simian virus 40 late transcription in vitro by a cellular factor binding the simian virus 40 21-base-pair repeat promoter element. Proc. Natl. Acad. Sci. USA 84: 6025-6029. http://dx.doi.org/10.1073/pnas.84.17.6025

Genetics and Molecular Research 16 (1): gmr16019479 
miR-660-5p in human breast cancer

Krishnan P, Ghosh S, Wang B, Li D, et al. (2015). Next generation sequencing profiling identifies miR-574-3p and miR660-5p as potential novel prognostic markers for breast cancer. BMC Genomics $16: 735$. http://dx.doi.org/10.1186/ s12864-015-1899-0

Lee YS and Dutta A (2007). The tumor suppressor microRNA let-7 represses the HMGA2 oncogene. Genes Dev. 21: 10251030. http://dx.doi.org/10.1101/gad.1540407

Li L, Yuan L, Luo J, Gao J, et al. (2013). MiR-34a inhibits proliferation and migration of breast cancer through downregulation of Bcl-2 and SIRT1. Clin. Exp. Med. 13: 109-117. http://dx.doi.org/10.1007/s10238-012-0186-5

Liu R, Chen X, Du Y, Yao W, et al. (2012). Serum microRNA expression profile as a biomarker in the diagnosis and prognosis of pancreatic cancer. Clin. Chem. 58: 610-618. http://dx.doi.org/10.1373/clinchem.2011.172767

Porter DA, Krop IE, Nasser S, Sgroi D, et al. (2001). A SAGE (serial analysis of gene expression) view of breast tumor progression. Cancer Res. 61: 5697-5702.

Saxena UH, Owens L, Graham JR, Cooper GM, et al. (2010). Prolyl isomerase Pin1 regulates transcription factor LSF (TFCP2) by facilitating dephosphorylation at two serine-proline motifs. J. Biol. Chem. 285: 31139-31147. http:// dx.doi.org/10.1074/jbc.M109.078808

Siegel R, Naishadham D and Jemal A (2013). Cancer statistics, 2013. CA Cancer J. Clin. 63: 11-30. http://dx.doi. org/10.3322/caac. 21166

Sørlie T, Perou CM, Tibshirani R, Aas T, et al. (2001). Gene expression patterns of breast carcinomas distinguish tumor subclasses with clinical implications. Proc. Natl. Acad. Sci. USA 98: 10869-10874. http://dx.doi.org/10.1073/ pnas. 191367098

Taylor MA, Sossey-Alaoui K, Thompson CL, Danielpour D, et al. (2013). TGF- $\beta$ upregulates miR-181a expression to promote breast cancer metastasis. J. Clin. Invest. 123: 150-163. http://dx.doi.org/10.1172/JCI64946

Umetani N, Giuliano AE, Hiramatsu SH, Amersi F, et al. (2006). Prediction of breast tumor progression by integrity of free circulating DNA in serum. J. Clin. Oncol. 24: 4270-4276. http://dx.doi.org/10.1200/JCO.2006.05.9493

$\mathrm{Xu} \mathrm{X,} \mathrm{Liu} \mathrm{Z,} \mathrm{Zhou} \mathrm{L,} \mathrm{Xie} \mathrm{H,} \mathrm{et} \mathrm{al.} \mathrm{(2015).} \mathrm{Characterization} \mathrm{of} \mathrm{genome-wide} \mathrm{TFCP2} \mathrm{targets} \mathrm{in} \mathrm{hepatocellular} \mathrm{carcinoma:}$ implication of targets FN1 and TJP1 in metastasis. J. Exp. Clin. Cancer Res. 34: 6. http://dx.doi.org/10.1186/s13046$\underline{015-0121-1}$

Genetics and Molecular Research 16 (1): gmr16019479 